\title{
BMJ Open Feasibility and acceptability of telehealth coaching to promote healthy eating in chronic kidney disease: a mixed-methods process evaluation
}

\author{
Jaimon T Kelly, ${ }^{1}$ Molly M Warner, ${ }^{1}$ Marguerite Conley, ${ }^{2}$ Dianne P Reidlinger, ${ }^{1}$ \\ Tammy Hoffmann, ${ }^{3}$ Jonathan Craig, ${ }^{4,5}$ Allison Tong, ${ }^{5,6}$ Marina Reeves, ${ }^{7}$ \\ David W Johnson, ${ }^{8,9,10}$ Suetonia Palmer, ${ }^{11}$ Katrina L Campbell ${ }^{1,2}$
}

To cite: Kelly JT, Warner MM, Conley M, et al. Feasibility and acceptability of telehealth coaching to promote healthy eating in chronic kidney disease: a mixed-methods process evaluation. BMJ Open 2019;9:e024551. doi:10.1136/ bmjopen-2018-024551

- Prepublication history and additional material for this paper are available online. To view these files, please visit the journal online (http://dx.doi org/10.1136/bmjopen-2018024551).

Received 3 June 2018 Revised 5 November 2018 Accepted 13 December 2018

Check for updates

(C) Author(s) (or their employer(s)) 2019. Re-use permitted under CC BY-NC. No commercial re-use. See rights and permissions. Published by BMJ.

For numbered affiliations see end of article.

Correspondence to Dr Jaimon T Kelly; jkelly@bond.edu.au

\section{ABSTRACT}

Objective To evaluate the feasibility and acceptability of a personalised telehealth intervention to support dietary self-management in adults with stage 3-4 chronic kidney disease (CKD).

Design Mixed-methods process evaluation embedded in a randomised controlled trial.

Participants People with stage 3-4 CKD (estimated glomerular filtration rate $[\mathrm{eGFR}] 15-60 \mathrm{~mL} / \mathrm{min} / 1.73 \mathrm{~m}^{2}$ ).

Setting Participants were recruited from three hospitals in Australia and completed the intervention in ambulatory community settings.

Intervention The intervention group received one telephone call per fortnight and 2-8 tailored text messages for 3 months, and then 4-12 tailored text messages for 3 months without telephone calls. The control group received usual care for 3 months then non-tailored education-only text messages for 3 months.

Main outcome measures Feasibility (recruitment, nonparticipation and retention rates, intervention fidelity and participant adherence) and acceptability (questionnaire and semistructured interviews).

Statistical analyses performed Descriptive statistics and qualitative content analysis.

Results Overall, 80/230 (35\%) eligible patients who were approached consented to participate (mean \pm SD age $61.5 \pm 12.6$ years). Retention was $93 \%$ and $98 \%$ in the intervention and control groups, respectively, and $96 \%$ of all planned intervention calls were completed. All participants in the intervention arm identified the tailored text messages as useful in supporting dietary selfmanagement. In the control group, 27 (69\%) reported the non-tailored text messages were useful in supporting change. Intervention group participants reported that the telehealth programme delivery methods were practical and able to be integrated into their lifestyle. Participants viewed the intervention as an acceptable, personalised alternative to face-face clinic consultations, and were satisfied with the frequency of contact.

Conclusions This telehealth-delivered dietary coaching programme is an acceptable intervention which appears feasible for supporting dietary self-management in stage 3-4 CKD. A larger-scale randomised controlled trial is needed to evaluate the efficacy of the coaching programme on clinical and patient-reported outcomes.
Strengths and limitations of this study

- This study used a pragmatic design which enhanced its feasibility.

- Mixed-methods captured both quantitative and qualitative data to determine multiple aspects of feasibility and acceptability.

- Interview data to determine the intervention's acceptability were not captured in control group participants.

Trial registration number ACTRN12616001212448; Results.

\section{INTRODUCTION}

Chronic kidney disease (CKD) is a progressive condition affecting over $10 \%$ of the population worldwide. ${ }^{1}$ The management of CKD is burdensome for patients, families and the healthcare system. With the incidence of end-stage kidney disease (ESKD) growing, there is a pressing need for preventative action. ${ }^{2}$ This includes the provision of pragmatic, person-centred interventions to support dietary behaviour change.

Diet is a modifiable risk factor for the progression of CKD to ESKD. ${ }^{3} 4$ Typical dietary advice given to people with CKD includes restricting individual nutrients, such as sodium, protein, potassium and phosphate. However, there is little evidence regarding the adherence to, and efficacy of, nutrient-specific dietary advice in CKD populations..$^{5}$ Recent evidence suggests that following a healthy dietary pattern, as a whole food-based dietary pattern is associated with a reduced risk of death in people with CKD. ${ }^{6}$ A focus on foods rather than single nutrients may also facilitate increased adherence to dietary change in people with $\operatorname{CKD}^{6} 7$ 
which is otherwise challenging due to dietary complexity and competing demands of other medical and lifestyle self-management. ${ }^{8}$ Overcoming these challenges to implementing sustained dietary change is necessary to test whether improving diet quality alters patient-centred outcomes.

Providing regular and individualised dietary support required for those with CKD comes with geographical, time and financial barriers. ${ }^{9}$ Furthermore, addressing diet quality requires more frequent and repetitive support that most health services are unable to provide. To determine whether increasing diet quality (through dietary pattern) may attenuate the progression of CKD and elevated cardiovascular risk on a sufficient scale for a randomised controlled trial (RCT), alternative modalities that are effective in supporting dietary management are needed. Telehealth modalities, particularly telephone-based and text message coaching, present an opportunity to overcome barriers and challenges that people with CKD encounter in accessing healthcare services. ${ }^{810}$ Telehealth interventions may facilitate an increased frequency and quality of contact between the patient and healthcare professional ${ }^{11} 12$ which may improve acceptability, uptake and adherence to interventions $^{13}$ and better align with a patient-centred model of care and reflect the needs of people with CKD. ${ }^{10}$ While clinical trials of telehealth-delivered dietary interventions conducted specifically in CKD are lacking, trials conducted in the broader chronic disease population have shown telehealth-delivered dietary interventions are effective at supporting behaviour change to reduce chronic disease risk, including improving diet quality, fruit and vegetable consumption and reducing dietary sodium intake, compared with face-to-face modalities. ${ }^{11}$ This may be due to the flexibility that both telephone and text messaging interventions provide in time and location, and the opportunity to offer more intensive dietary coaching that may not be feasible with traditional care models. ${ }^{14-16}$ Text messaging has been used to 'extend contact' after an intervention and has been shown to maintain clinical outcomes and minimise intervention decay. ${ }^{17}{ }^{18}$ A systematic review of text message health interventions highlighted the need for better evidence on the relative effectiveness of text-based interventions including the level of tailoring of text message delivery (incorporating frequency and timing), level of interaction (ie, response and feedback) and impact of additional interventions (such as a combination with telephone, face-to-face, video or internet). ${ }^{19}$

While dietary patterns aligned with a higher diet quality are associated with lower mortality in $\mathrm{CKD},{ }^{6}$ the level of tailoring and individualised coaching required to achieve and support dietary self-management is unknown. Non-CKD trials have demonstrated effectiveness for minimally tailored text messages, ${ }^{20}$ information-only text messages and tailored interactive text messages. ${ }^{21}$ However, no approach has been shown to be superior and no study has investigated such questions in the CKD population. To determine the level of tailoring, and the delivery method that is most feasible and acceptable for patients with CKD, this pilot study aimed to evaluate the feasibility and acceptability of telehealth-delivered dietary coaching to support dietary self-management in stage 3-4 CKD.

\section{MATERIALS AND METHODS}

We used a mixed-methods design in this pilot study, whereby qualitative data on the patient experiences were embedded within quantitative data relating to participants recruited into the Evaluation of iNdividualized Telehealth Intensive Coaching to promote healthy Eating and lifestyle in Chronic Kidney Disease (ENTICE-CKD) programme. All data were prospectively collected. This pilot RCT was prospectively registered and reported based on the extension of the Consolidated Standards of Reporting Trials statement for feasibility and pilot studies. $^{22}$

\section{Design}

This mixed-methods process evaluation was embedded in an RCT, conducted from November 2016 to November 2017. The dietary intervention was designed using the social cognitive theory, ${ }^{23}$ with a patient-centred focus on improving self-management to reduce dietary sodium intake $(<2300 \mathrm{mg} /$ day $)$ and increase dietary quality in accordance with the Australian Dietary Guidelines (see online supplementary table 1 for intervention guidance) ${ }^{24}$ The constructs of the social cognitive theory most used were outcome expectation (through education text messages and calls), self-regulation (through goal setting, self-monitoring, coaches' feedback during calls and text-message goal-check replies) and self-efficacy (through setting small, achievable goals, celebrating success, encouraging self-monitoring and prompting problem solving in calls and text messages). Interventions were adjunct to usual nephrology care from treating physician(s) and renal team members, including ad hoc referrals to allied health practitioners during the study.

\section{Participants}

Participants were recruited from three tertiary nephrology units in Queensland, Australia over a 6-month period. Inclusion criteria were: adults over 18 years of age; stage 3-4 CKD (eGFR $15-60 \mathrm{~mL} / \mathrm{min} / 1.73 \mathrm{~m}^{2}$ ); and access to a mobile device capable of receiving text messages and telephone calls. Exclusion criteria were: anticipated dialysis commencement or kidney transplant within the following 12 months, pregnancy, non-English speaking, cognitively impaired or deemed unfit to participate by their treating nephrologist.

Potential participants were screened for eligibility by a local site investigator or research nurse from daily outpatient appointment lists and relevant hospital databases. Following discussion with their treating nephrologist, people were approached and invited to participate. If 
people were unable to be contacted at their outpatient appointment, they were mailed a written invitation to participate with a phone number to contact if they were interested.

Eligible participants were randomised on a 1:1 ratio into one of two groups (stratified by recruiting site (site A, $\mathrm{B}, \mathrm{C}$ ) and presence of diabetes (yes, no) in blocks of 8's). Randomisation was completed by computer-generated random numbers carried out by an independent statistician not involved in the study.

\section{Study treatment}

The ENTICE-CKD programme was completed in two 3-month phases in both the intervention and control groups of the study as outlined in online supplementary figure 1 and the details of the intervention according to the template for intervention description and replication (TIDieR) items $(1-10)^{25}$ is described in online supplementary table 2. Details about the intervention fidelity TIDieR items (11 and 12) is described and reported throughout this paper and is not summarised in online supplementary table 2. Each participant was involved in the trial for six consecutive months. All participants were provided with an ENTICE-CKD workbook at the baseline visit. The 90-page workbook included information on setting specific, measurable, achievable, realistic and timebound goals; eating well for kidneys (based on the Australian Dietary Guidelines; online supplementary table 1$)^{24}$; active living (based on the Australian Physical Activity Guidelines) ${ }^{26}$; role of diet in kidney disease, strategies for planning, self-monitoring checklists and a list of useful websites, apps and recipes for further reference.

\section{Telehealth coaches}

Each participant was assigned to one of two telehealth coaches at baseline. The participant had the same coach for the duration of the programme. Both telehealth coaches were registered dietitians (Australian equivalent) with additional training in renal nutrition, behaviour change and motivational interviewing; were external to the recruiting sites and had never met the participants; and were not involved in any outcome data collection.

\section{Phase 1}

The participants in the intervention group received six fortnightly telephone calls in phase 1 which were scheduled on weekdays at a time of the participants choosing (from 07:00 hours to 19:00 hours). The first call was scheduled for $45 \mathrm{~min}$ and five subsequent calls for approximately $30 \mathrm{~min}$. Each call was based on established protocols and call scripts. The telephone call content was guided by the workbook topics, structured according to the 5A's framework (Assess, Advise, Agree, Assist, Arrange), ${ }^{27}$ and individually tailored to participants using relevant educational strategies, and in consideration of the participant goals and comorbidities. Where required, 24 hours dietary recalls were undertaken during coaching calls to track adherence and progress with goals. Coaches used Microsoft Excel $^{28}$ to document progress of each call and log information including goal setting, implementation intentions, self-monitoring tools, call attempts and durations, and text message preferences.

In addition, participants in the intervention group received two to eight text messages scheduled between coaching calls with the actual number and time of day determined by each participant's preference. Text categories included: educational, self-monitoring and goal setting. The schedule of text messages for the intervention and control group in phases 1 and 2 is detailed in online supplementary table 2 . The text messages were sent using a web-based, semiautomated text message management platform (Propelo, www.propelo.com.au), developed and administered by The University of Queensland's School of Public Health. ${ }^{29}$ The investigators, in consultation with local nephrologists, dietitians and evidence-based practice guidelines, designed the library of text messages which were then reviewed for comprehension by a group of patients, nephrologists and members of the investigator team. The text message library was imported into the software platform which was designed to tailor text messages based on: participant's name, individual goals, barriers to achieving goals and participant-identified solutions to overcoming those barriers. These tailoring variables were collected and modified as required by the coaches following the initial and subsequent coaching calls.

As shown in online supplementary table 2, participants in the intervention group could receive one 'goal check' per goal (total two goal checks) per fortnight in phase 1 and up to two goal checks per goal (total 2-4 goal checks) per fortnight in phase 2 . These goal checks required the participant to respond to the text with a 'yes' or 'no' which prompted the software to send a predetermined response. An incoming text reply outside protocol (ie, not a 'yes' or 'no' response) was classified as an 'unrecognised response'. This triggered an email to the participant's coach and was only responded to where participants expressed considerable risk to their health (eg, symptoms needing medical attention).

Participants in the control group received no coaching or text messages between the baseline visit and 3 months (phase 1). The control group continued to receive standard care under their treating nephrologist (typically one clinic visit every 3 months) and were encouraged to work through the ENTICE-CKD workbook at their own pace.

\section{Phase 2}

At 3 months, participants in the intervention group completed a tailoring telephone call with their coach to determine individual preferences for the timing and frequency of text messages for phase 2. At 18 weeks (ie, half way through phase 2), participants received a second tailoring call where they could modify the timing and frequency of text messages and could update their goals. Intervention group participants chose text message frequencies (4-12 text messages per fortnight) for the 
same types of texts that they received in phase 1 (educational tips, self-monitoring, goal checks). Participants in the control group received non-tailored education-only text messages (described in online supplementary table 2 ) at the commencement of phase 2 of the trial. This intervention was additional to the usual care participants in the control group were receiving in phase 1.

\section{Data collection}

Basic demographic data (including participant's age and gender) were recorded at baseline. Socioeconomic status was estimated from participants' postcodes, according to the Index of Relative Socioeconomic Advantage and Disadvantage. ${ }^{30}$ Baseline health literacy was collected using the single-item Literacy Screener which classifies health literacy as good or limited based on the single question, 'How often do you need to have someone help you when you read instructions, pamphlets or other written material from your doctor or pharmacy? 31

\section{Reach and retention}

The sample size was determined for the purpose of informing a future study. Therefore, a target of 30-40 participants per arm was set to allow for meaningful and reliable data which could be used to power future trials. ${ }^{32}$ Recruitment and non-participation rates were captured across the three recruitment sites, with a goal to achieve the target sample size of 80 participants in the 6-month recruitment time frame. Retention rate was measured at three and 6 months in both study groups, with successful retention defined $80 \%$ at the 6-month study end.

\section{Intervention delivery}

Individual cases were discussed fortnightly between the coaches and the lead investigator to support consistent intervention delivery. All coaching calls were audio recorded, from which $10 \%$ were assessed for consistency by peer-review by an individual external to the project. Consistency considered the predefined call scripts and potential deviation from the call scripts with reasons for why this occurred. The following fidelity data were also collected and stored in a Microsoft Excel $^{28}$ database throughout the trial: number, duration and content of coaching telephone calls; number and type of text messages delivered; number and type of text message responses; and time spent by coaches for each interaction.

\section{Intervention adherence}

Adherence was defined as successfully completing five of the six telephone calls for the intervention group. Data were also collected on individual participant adherence to the dietary intervention, collected by coaches in each telephone call using a call log template in Microsoft Excel. ${ }^{28}$ In the call logs, coaches described evidence of the participant's overall progress, evidence of self-monitoring, goals set and implementation intentions (behaviours implemented to achieve goals) during each call which was quantified in counts to capture participant adherence.
Acceptability

A utility and acceptability survey of the text message component of the ENTICE-CKD trial was collected from all participants at the 6-month end of study visit (online supplementary table 3). The survey included 13 items, developed specifically for the study, with five items asking participants to rate on a 5-point Likert scale from 1 'strongly disagree' to 5 'strongly agree', four items asking participants yes/no questions and four multiple choice questions, based on previous methodology in patients with cardiac disease. ${ }^{20}$ In addition to this, during the sixth telephone call (3-month study mid-point; for intervention participants only), coaches obtained verbal consent of participants to be approached to complete an interview relating to their experiences of the intervention.

Semistructured interviews were conducted in-person and by telephone. Participants were recruited based on consecutive sampling of completing participants until data saturation was achieved. The interviews were conducted by investigator (MW), who had not previously met the participants and was not involved in the planning of the intervention. The interview guide included questions on: barriers and facilitators of programme adherence; telehealth delivery methods and frequency of contact; usability of the programme; goal setting, self-monitoring, behaviour change; and experiences (online supplementary table 4). Modification of the interview guide occurred after each interview to broaden scope of the data collected. Interviews were audio-recorded and transcribed verbatim.

\section{Patient involvement}

The study was designed in collaboration with similar participants as those recruited for this study. This patient engagement was conducted as a qualitative study, reported elsewhere by the investigators ${ }^{10}$ and details the patient reported burden associated with following dietary recommendations that were considered while developing this trial. All intervention materials, including the workbook and text messages, were reviewed by people with CKD with feedback forms which were used to revise all the material before production. No patients were involved in the recruitment or data collection of this process evaluation study. A summary of the main results will be mailed out to participants. The burden of the trial has been evaluated in semistructured interviews, and will be reported elsewhere.

\section{Data analysis}

Quantitative data were analysed using simple descriptive statistics (counts and percentages). To determine the difference in the utility and acceptability between the two study groups, a standard $\chi^{2}$ test was used with a significance level determined as $\mathrm{p}<0.05$. Statistics were conducted in SPSS Statistics for Windows (V.22.0) and Microsoft Excel. ${ }^{28}$

Inductive content analysis ${ }^{33}$ of the semistructured interview transcripts regarding acceptability of the 
intervention was conducted researcher (MW) who was not involved in quantitative data planning, collection and analysis. After familiarisation with the data, an open coding approach was adopted to identify, develop and finalise categories and subcategories within the data. A dietitian and qualitative researcher (DR) familiar with the data then finalised and confirmed emerging categories that were relevant to the process evaluation. Verbatim quotes were collected and used to represent attributes demonstrated for both the feasibility and acceptability of the ENTICE-CKD programme. Microsoft Word ${ }^{34}$ was used to facilitate data management (tables) and basic content analysis (comments relating to attributes demonstrating feasibility and acceptability) of data.

\section{RESULTS}

\section{Characteristics of participants}

The baseline characteristics of the participants are reported in table 1 . Of the 80 participants who completed their baseline visit, $64 \%$ were men and had a mean age of 62 years. The stage of CKD varied within the sample, with $31 \%$ stage 3 a $\left(\right.$ eGFR $\left.45-59 \mathrm{~mL} / \mathrm{min} / 1.73 \mathrm{~m}^{2}\right), 44 \%$ stage $3 \mathrm{~b}$ (eGFR $30-44 \mathrm{~mL} / \mathrm{min} / 1.73 \mathrm{~m}^{2}$ ) and $25 \%$ stage 4 (eGFR $15-29 \mathrm{~mL} / \mathrm{min} / 1.73 \mathrm{~m}^{2}$ ). The most common comorbidities were hypertension $(81 \%)$ and diabetes $(39 \%)$ (table 1). Baseline health literacy was good in over $90 \%$ of all participants. Baseline characteristics were well balanced across the two groups, suggesting randomisation was effective.

\section{Reach and retention}

Participants were recruited between November 2016 and May 2017, from Gold Coast (43\%), Sunshine Coast $(31 \%)$ and Brisbane $(26 \%)$ hospitals. The flow of participants through the ENTICE-CKD study is shown in figure 1. A total of 230 potentially eligible individuals were approached and invited to participate, of whom 80 participants $(35 \%)$ were recruited to the ENTICE-CKD trial. Of the 146 individuals who declined to participate, 'not interested' was the most commonly stated reasons for non-participation $(36 \%)$ followed by perceived excessive time commitment (16\%), having other medical conditions which are taking priority (13\%), travel burden to make study visits $(11 \%)$ and already feeling healthy $(10 \%)$. Other reasons for non-participation included already seeing a dietitian $(6 \%)$, believed the intervention did not fit their current lifestyle $(6 \%)$ or preferred not to use technology (1\%). A further two individuals $(1 \%)$ consented to the study but did not attend a baseline visit and were therefore not randomised to a treatment group.

Seventy-six $(95 \%)$ of all randomly allocated participants completed the 6-month telehealth programme. A total of four $(5 \%)$ participants withdrew from the study. All the withdrawals occurred in the first 3 months of the programme. Three of the four participants who withdrew were from the intervention group (two were unable to be contacted and therefore did not commence the
Table 1 Demographics of participants whom completed the 6-month ENTICE-CKD pilot study

\begin{tabular}{|c|c|c|}
\hline Characteristic & $\begin{array}{l}\text { Intervention } \\
\text { group }(n=41)\end{array}$ & $\begin{array}{l}\text { Control } \\
\text { group }(n=39)\end{array}$ \\
\hline Male, n (\%) & $26(63)$ & $25(64)$ \\
\hline Age (years) & $62.0 \pm 12.0$ & $61.1 \pm 13.3$ \\
\hline \multicolumn{3}{|c|}{ Stage of chronic kidney disease, n (\%) } \\
\hline $3 a$ & $10(25)$ & $15(38)$ \\
\hline $3 b$ & $19(46)$ & $16(41)$ \\
\hline 4 & $12(29)$ & $8(21)$ \\
\hline Body mass index, $\mathrm{kg} / \mathrm{m}^{2}$ & $33.4 \pm 6.7$ & $31.0 \pm 6.4$ \\
\hline Hypertension, n (\%) & $34(83)$ & $31(80)$ \\
\hline Diabetes, n (\%) & $15(37)$ & $16(41)$ \\
\hline Active smoker status, n (\%) & $21(51)$ & $16(41)$ \\
\hline \multicolumn{3}{|l|}{ Ethnicity, n (\%) } \\
\hline Asian & $2(5)$ & $1(3)$ \\
\hline Caucasian/European & $37(91)$ & $32(82)$ \\
\hline Indigenous & $1(2)$ & 0 \\
\hline Other & $1(2)$ & $6(15)$ \\
\hline \multicolumn{3}{|l|}{ Education, $\mathrm{n}(\%)$} \\
\hline Lower than 10th grade & $17(42)$ & $12(32)$ \\
\hline Up to 12 th grade & $4(10)$ & $10(26)$ \\
\hline Tertiary educated & $20(47)$ & $16(41)$ \\
\hline \multicolumn{3}{|c|}{ Socioeconomic status, n (\%) } \\
\hline High & $27(66)$ & $25(64)$ \\
\hline \multicolumn{3}{|l|}{ Health literacy, n (\%) } \\
\hline Good & $37(90)$ & $36(92)$ \\
\hline
\end{tabular}

ENTICE-CKD, Evaluation of iNdividualized Telehealth Intensive Coaching to promote healthy Eating and lifestyle in Chronic Kidney Disease.

programme, and one participant was unable to continue due to a family illness). The sole participant who withdrew from the control group did not report a reason for doing so. There were no appreciable differences in the demographics of those participants who dropped out compared with those remaining in the trial.

\section{Intervention delivery}

Table 2 shows the adherence to the planned delivery of the telephone and text message components of the ENTICE-CKD intervention. The delivery of the scheduled telephone calls was conducted according to protocol with $90 \%$ of planned calls being completed as scheduled. The mean duration of the first intervention call was $45.5 \pm 10 \mathrm{~min}$ (range $28-75 \mathrm{~min}$ ). The mean length of the subsequent five calls was $24 \pm 10 \mathrm{~min}$ (range 2-62 $\mathrm{min}$ ).

A total of 4985 intervention text messages were sent to ENTICE participants. The median number of text messages sent to participants was within protocol for both groups, with intervention participants receiving a 


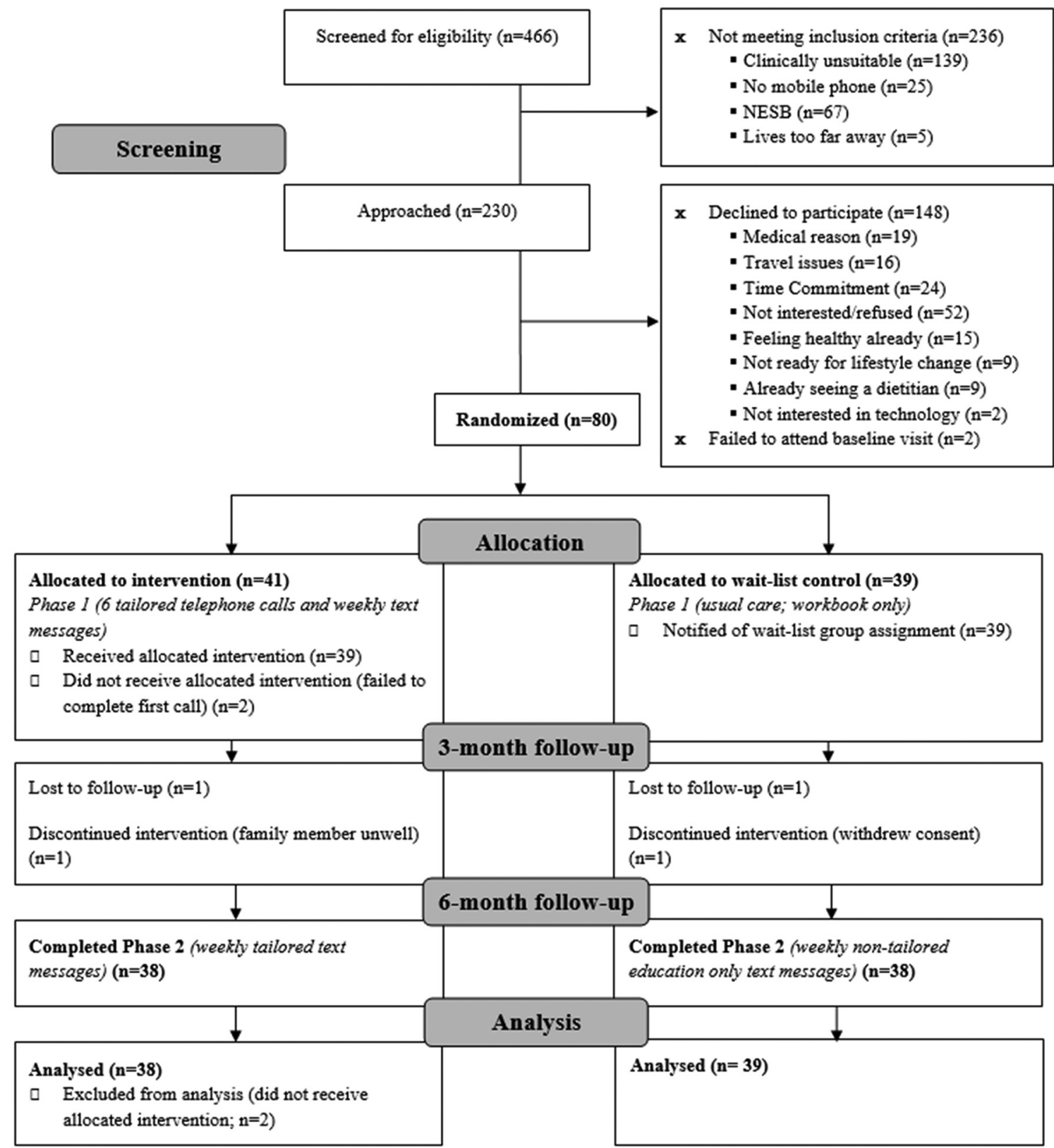

Figure 1 Consolidated Standards of Reporting Trials flow diagram showing the flow of participants through the Evaluation of iNdividualized Telehealth Intensive Coaching to promote healthy Eating and lifestyle in Chronic Kidney Disease study.

median of four text messages per fortnight in phase 1 and seven per fortnight in phase 2. Control participants received a median of six non-tailored education-only text messages per fortnight in phase 2 (table 2). The total number of incoming text messages (replies from participants) was 1100 (table 2), 36\% ( $\mathrm{n}=400)$ triggered the appropriate goal-check reply, $3 \%(n=31)$ required the dietitian coach to send a tailored text message to address the concern raised by the sender and $61 \%$ $(\mathrm{n}=669)$ required no reply.

\section{Intervention adherence}

A total of 38 participants (95\%) completed at least five calls, and $36(90 \%)$ completed all six calls. Two participants (5\%) never received a telephone call. Goal setting was completed by all participants in the first call as planned, with $95 \%$ of the participants setting two or more goals. The coaches' call logs showed that, throughout the programme, participants continued setting new goals with $10(26 \%)$ updating at least one goal in call two and $22(61 \%)$ updating at least one goal throughout the remaining four calls (table 3). A total of $29(76 \%)$ participants showed evidence of self-monitoring by the second call which was sustained throughout phase 1 of the intervention. Evidence of implementation intentions indicated that the majority of participants $(82 \%)$ needed at least two calls to begin putting planned dietary intentions in place. This number continued to rise 
Table 2 Delivery and response of fortnightly telephone calls and text messages in ENTICE-CKD

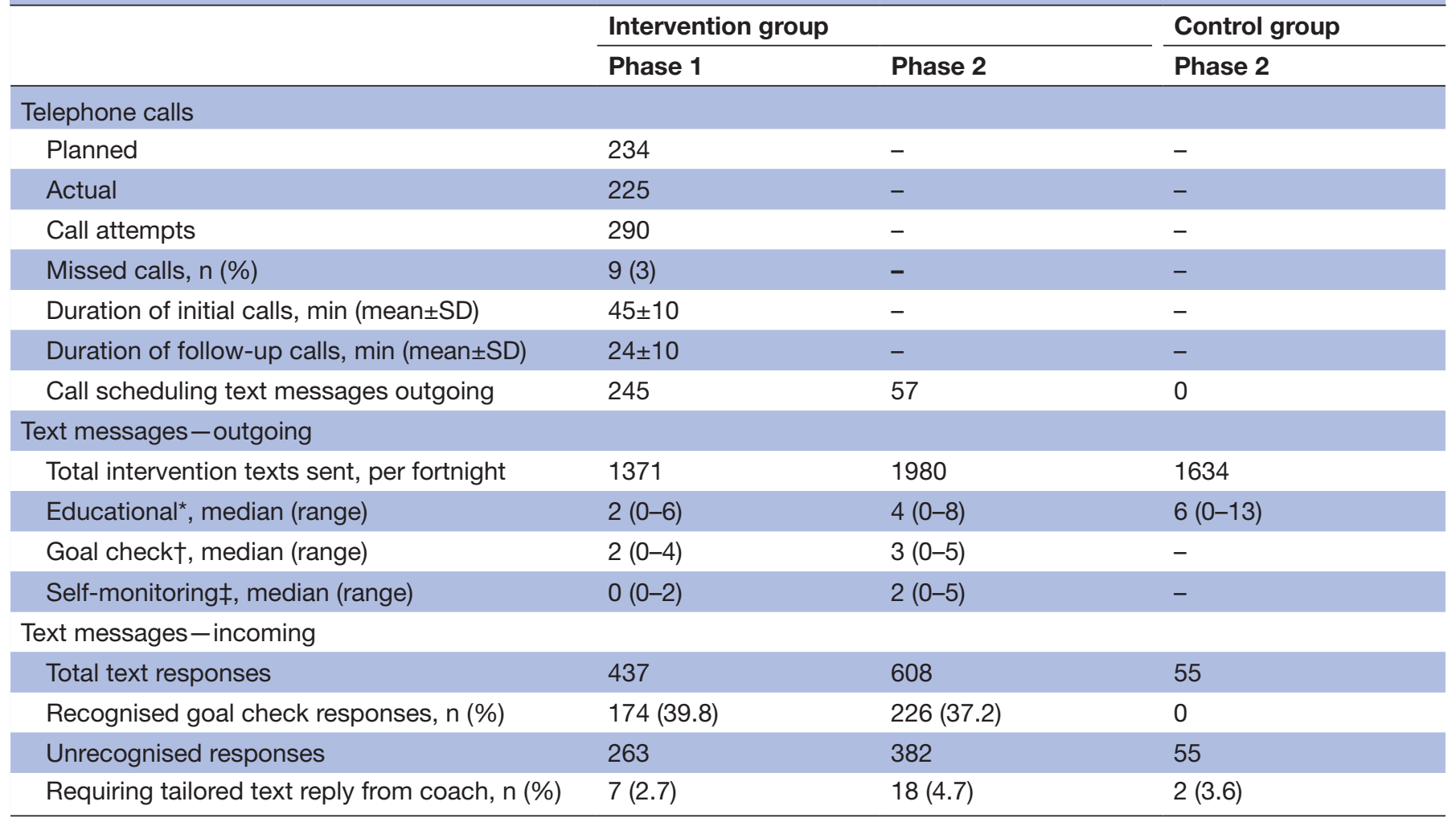

*Outcome expectations (providing information on consequence).

†Self-regulation.

$\ddagger$ Self-regulation (facilitate planned behaviour change).

ENTICE-CKD, Evaluation of iNdividualized Telehealth Intensive Coaching to promote healthy Eating and lifestyle in Chronic Kidney Disease.

over the next four calls to $97 \%$ by the end of phase 1 of the intervention.

\section{Acceptability}

\section{Utility and acceptability}

There were several differences in ratings for utility and acceptability between the intervention (tailored-text) group compared with the non-tailored education-only text message (control) group (table 4). Participants agreed (responses for 'agree' and 'strongly agree') that the text message component: supported their dietary self-management (intervention 100\%; 69\% control, $\mathrm{p}=0.003)$; provided motivation to change their diet (intervention $75 \%$, control $50 \%$; $\mathrm{p}=0.03$ ); and led them to a healthier diet (intervention $81 \%$, control $61 \%$, $\mathrm{p}=0.06$ ). There were no other differences observed in the utility of the text messages between the groups. The majority of text messages were saved and not deleted ( $77 \%$ overall), and $62 \%$ were shared with family, friends or healthcare providers across the two study groups. Acceptability of the text messages was assessed as high with $78 \%$ of all intervention and control participants reporting that the characteristics of the text messages (language, frequency, programme length, time of delivery) were satisfactory.
Attributes of feasibility and acceptability

Twenty-one intervention participants were interviewed on completion of phase 1 , either by telephone $(n=20)$ or face-to-face $(n=1)$. Interviews ranged from 20 to $96 \mathrm{~min}$ (mean $49 \mathrm{~min}$ ). Overall, participants had positive experiences with the ENTICE-CKD trial. Attributes of the discussions are described in nine categories within components of acceptability and feasibility (table 5). The acceptability categories discussed by participants were: acceptable alternative to clinic, preference for voice communication, regular contact via text message and personalised messages valued. The categories described under feasibility were: programme integrated into lifestyle, diverse delivery modes, social accountability, responding to dietary advice and infeasible elements beyond intervention. Participants emphasised the importance of social accountability; all participants expressed benefit from the relationship built with their coach. Participants identified benefits from telehealth delivery of the intervention, with the majority expressing preference for telehealth over face-to-face interventions. They appreciated the personable, bidirectional conversation of the telephone calls. The degree of usefulness of text messages was rated with some variability, although no participants described the content or delivery of text messages negatively in the semistructured interviews. The only areas of variability 


\begin{tabular}{|c|c|c|c|}
\hline Adherence & Call 1 & Call 2 & Call 3-6 \\
\hline Total planned calls & 39 & 39 & 156 \\
\hline Calls delivered & $39(100)$ & $38(97)$ & $148(95)$ \\
\hline Number of missed calls & 0 & $1(3)$ & $8(5)$ \\
\hline Due to withdrawal from trial & & & $2(1)$ \\
\hline Due to travel & & & $2(1)$ \\
\hline Other* & & $1(3)$ & $4(3)$ \\
\hline Goal setting & $38(100)$ & $10(26)$ & $23(61)$ \\
\hline 1 goals & $2(5)$ & $8(21)$ & $12(32)$ \\
\hline 2 goals & $36(95)$ & $2(5)$ & $7(18)$ \\
\hline 3 goals & N/A† & N/A† & $1(3)$ \\
\hline 4 goals & N/A† & N/A† & $3(8)$ \\
\hline Self-monitoring & $22 / 38(58)$ & 29/38 (76) & $29 / 38(76)$ \\
\hline \multicolumn{4}{|l|}{ Implementation intentions } \\
\hline Yes & $14(37) \ddagger$ & $31(82)$ & $37(97)$ \\
\hline No & $24(63) \ddagger$ & $7(18)$ & $1(3)$ \\
\hline
\end{tabular}

Data are presented as $\mathrm{n}(\%)$.

*One participant decided to get tailored text messages only following call 1.

†N/A: Not Applicable - In each call, only two goals could be set or updated.

‡Implementation intentions were not expected to be evident in the first call.

ENTICE, Evaluation of iNdividualized Telehealth Intensive Coaching to promote healthy Eating and lifestyle.

were noted in the small number of participants who were not familiar with using text messaging in their everyday life. Messages that were perceived to be personalised were preferred for both calls and text messages. Participants felt that receiving information via more than one delivery mode was helpful for making diet changes. Some participants discussed challenges which were not addressed by the ENTICE-CKD intervention, such as participants not being easily able to implement routine dietary behaviours while travelling, or those lacking social support outside of the programme.

\section{DISCUSSION}

This mixed-methods process evaluation study within an RCT evaluated the feasibility and acceptability of the ENTICE-CKD telehealth coaching programme to promote healthy eating among people with moderate CKD. The ENTICE-CKD programme was a feasible intervention that was delivered according to protocol and enabled participant adherence. The tailored telephone calls and text messages were acceptable to intervention participants in this pilot. In contrast, the acceptability varied for those in the non-tailored education-only text message (control) group. The ENTICE-CKD programme made participants feel supported and motivated for dietary self-management. However, this was more strongly indicated by participants who received the tailored intervention programme, as opposed to the control group
Table 4 Utility and acceptability of ENTICE-CKD text messages by participant group*

\begin{tabular}{|c|c|c|}
\hline Characteristic & $\begin{array}{l}\text { Tailored } \\
\text { text } \\
\text { messages }\end{array}$ & $\begin{array}{l}\text { Non-tailored } \\
\text { text messages }\end{array}$ \\
\hline \multicolumn{3}{|l|}{ Usefulness and understanding } \\
\hline $\begin{array}{l}\text { Q1: Useful in supporting } \\
\text { dietary change }\end{array}$ & $100 \%$ & $69 \%$ ** \\
\hline $\begin{array}{l}\text { Q2: Messages were easy to } \\
\text { understand }\end{array}$ & $100 \%$ & $100 \%$ \\
\hline \multicolumn{3}{|c|}{ Influence on motivation and behaviour change } \\
\hline $\begin{array}{l}\text { Q3: Messages motivated } \\
\text { change }\end{array}$ & $75 \%$ & $50 \%$ ** \\
\hline $\begin{array}{l}\text { Q4: Healthier diet due to } \\
\text { messages }\end{array}$ & $81 \%$ & $61 \%$ \\
\hline $\begin{array}{l}\text { Q5: Exercise increased due } \\
\text { to messages }\end{array}$ & $38 \%$ & $33 \%$ \\
\hline \multicolumn{3}{|l|}{ Message saving and sharing } \\
\hline $\begin{array}{l}\text { Q6: Per cent of messages } \\
\text { read }\end{array}$ & $100 \%$ & $100 \%$ \\
\hline Q7: Saved messages & $81 \%$ & $72 \%$ \\
\hline Q8: Shared messages & $56 \%$ & $67 \%$ \\
\hline Family member & $71 \%$ & $74 \%$ \\
\hline Friend & $12 \%$ & $10 \%$ \\
\hline Health provider & $12 \%$ & $10 \%$ \\
\hline \multicolumn{3}{|c|}{ Appropriate message characteristics } \\
\hline Q9: Suitable language & $100 \%$ & $100 \%$ \\
\hline $\begin{array}{l}\text { Q10: Texts were not too } \\
\text { regular }\end{array}$ & $94 \%$ & $86 \%$ \\
\hline $\begin{array}{l}\text { Q11: Programme length } \\
\text { (6 months) }\end{array}$ & $88 \%$ & $78 \%$ \\
\hline $\begin{array}{l}\text { Q12: Appropriate time of } \\
\text { the day/night }\end{array}$ & $100 \%$ & $94 \%$ \\
\hline
\end{tabular}

${ }^{*}$ Response rate for this survey was 73 out of 80 participants (91\%), tailored text messages $(n=43)$, non-tailored text messages $(n=39)$. ${ }^{* *} \mathrm{P}<0.01$ between groups.

ENTICE-CKD, Evaluation of iNdividualized Telehealth Intensive Coaching to promote healthy Eating and lifestyle in Chronic Kidney Disease.

who received non-tailored education-only text messages. These results suggest that a tailored approach to text messaging may be important to people with CKD, as it may facilitate the support and regular interaction for dietary changes. ${ }^{8}$ Participants felt that the frequent contact via calls and text messages reinforced rapport and built a supportive relationship between participant and coach which, in turn, enabled stronger social accountability and progressive dietary change.

The successful recruitment and retention of participants enrolled in the ENTICE trial demonstrated feasibility. Although it is important to consider the trial only had a $35 \%$ recruitment rate, the feasibility was strengthened by the successful recruitment in the anticipated 
Table 5 Acceptability and feasibility of ENTICE-CKD programme at completion of phase 1 (intervention group): qualitative content analysis of semistructured interviews $(n=21)$

\begin{tabular}{|c|c|c|}
\hline Category & Attributes & Quote \\
\hline \multicolumn{3}{|l|}{ Acceptability } \\
\hline $\begin{array}{l}\text { Acceptable alternative } \\
\text { to clinic }\end{array}$ & $\begin{array}{l}\text { Overcomes clinic wait times, transport logistics. } \\
\text { Flexibility of phone call appointment times. } \\
\text { Preferred talking from a familiar environment and } \\
\text { not feeling rushed. } \\
\text { No identified disadvantages of telehealth } \\
\text { communication vs face-to-face. } \\
\text { Building rapport with coach. }\end{array}$ & $\begin{array}{l}\text { 'At home I'm more relaxed and I have the book in } \\
\text { front of me and I was able to jot down anything } \\
\text { that was important, if I was at the hospital there's } \\
\text { so many people around and you don't feel very } \\
\text { relaxed, you feel like everyone is listening to } \\
\text { your conversation, so you don't say personal } \\
\text { information' Female, } 69\end{array}$ \\
\hline $\begin{array}{l}\text { Preference for voice } \\
\text { communication }\end{array}$ & $\begin{array}{l}\text { More benefit from voice calls. } \\
\text { Frequency of fortnightly phone calls. }\end{array}$ & $\begin{array}{l}\text { 'I found the calls better than the texts...they were } \\
\text { more personable and kept me on track' Female, } 68\end{array}$ \\
\hline $\begin{array}{l}\text { Regular contact via text } \\
\text { message }\end{array}$ & $\begin{array}{l}\text { Text messages were an acceptable mode of } \\
\text { communicating information. } \\
\text { Preference for receiving text messages with } \\
\text { personal encouragement and general tips. } \\
\text { All text messages were acceptable. }\end{array}$ & $\begin{array}{l}\text { 'We solved a lot of my little issues, and it's given me } \\
\text { a lot better understanding, and you know the more } \\
\text { you think about it and communicate about it, ah the } \\
\text { better it is' Male, } 71\end{array}$ \\
\hline
\end{tabular}

Feasibility

Programme integrated Length of phone calls easily accommodated.

into lifestyle

- Twelve-week telephone intervention enough time for change.

Self-monitoring the behaviour of choice.

'As long as you're getting information backwards and forwards, that's the more important thing than the length of the call, it's what you're getting out of it' Male, 78

Diverse delivery modes Active learning from a range of understandable delivery modes.

- Hard copy workbook as reference tool.

- Receiving explanations develops understanding and awareness of reasons for dietary change.

- Quantifiable dietary recommendations (food groups, 'good vs bad' foods, portion sizes, sodium levels).

Social accountability $\quad$ Supportive relationship with one coach allows
progressive dietary change.
Frequent reminders and reinforcing goals.
Interaction with coach via text messages.

'You've got to eat these foods, food groups and that, but you don't actually know the right quantities...this program shows it to you and it's like, it's teaching someone how to walk again' Male, 46

'The book I think was brilliant, because you've got that to go back through all the time, well any time you're doubtful you've got thoughts, you just look at the book, I did, I still do it' Male, 64

'If I didn't have the phone calls from [my coach] once a fortnight I probably wouldn't have taken it as serious as I have' Male, 65

'The support, even just texting and that, it's still, you know someone's doing it. It's, it just makes you feel better as a person, to know someone cares' Male, 64

Responding to dietary advice

\section{Infeasible elements beyond intervention}

- Small changes at a time.

- Practical strategies, manipulating environment to support behaviours, skill development (label reading).

- Setting goals and finding satisfaction in quantifiable outcomes (eg, portion sizes, food group servings).

- Physical comorbidities a barrier for lifestyle component of programme.

- Lack of support from others with poor understanding or low interest.

- Unstable or unsupportive environment for creating healthy habits.
'The program is delivered in segments, you're just having a bit of information at a time, so it's not overwhelming' Female, 68

'I was astounded at the salt content of it all, so when I read that I immediately stopped all salt that I put on my plate... I've not had salt since, so that was 3 months ago' Male, 65

'I have just been moving around a lot more and not in a stable environment of being in familiar surroundings, being unable to replicate...the menus... due to my transient nature of where I am presently' Male, 46

ENTICE-CKD, Evaluation of iNdividualized Telehealth Intensive Coaching to promote healthy Eating and lifestyle in Chronic Kidney Disease. 
6 -month recruitment period and very low attrition rate $(5 \%)$ at 6 months. Attrition is a common problem in studies of self-management in CKD which is reported as between $11 \%$ and $39 \%$, and which reduces the generalisability of findings, particularly given the often underpowered sample sizes of trials of lifestyle interventions in $\mathrm{CKD}^{35}$

The intensive coaching intervention had a high call completion rate $(90 \%)$ and high intervention adherence. This is similar to the $90 \%$ call completion rates reported in other telehealth studies in weight management, ${ }^{36}$ breast cancer ${ }^{17}$ younger adults in the general population, ${ }^{37}$ and CKD studies. ${ }^{38}$ A study involving 436 participants with CKD in the UK, who received a combination of interactive web-based resources and telephone follow-up demonstrated successful recruitment, retention and intervention satisfaction. ${ }^{38}$ There was no specific dietary education provided to participants in that study, however the community support intervention, provided through a workbook, online portal and telephone follow-up demonstrated a $69 \%$ recruitment rate, and had $85 \%$ retention at the 6-month follow-up. Participants reported over $80 \%$ usefulness for the workbook, $62 \%$ for the telephone calls and $23 \%$ for the interactive website. ${ }^{38}$ Considering the limited evidence on lifestyle interventions in CKD specifically, the findings from this trial support the feasibility of using telehealth coaching to support dietary self-management of CKD. The major difference between the study conducted by Blakeman and colleagues ${ }^{38}$ and the ENTICE-CKD study was that recruitment occurred in general practices compared with tertiary hospitals in our study. Our patient-engagement work highlighted the desire of people with CKD for preventative diet and lifestyle advice in the early stages of CKD, before it became a clinical issue. ${ }^{10}$ This possibly explains the higher recruitment rate in the primary care study by Blakeman and colleagues $(69 \%)$ compared with our study in the tertiary hospital setting (35\%).

Overall, there is limited evidence on the acceptability of telehealth dietary interventions in CKD ${ }^{39}$ A pilot study in 47 CKD participants demonstrated over $80 \%$ user adherence and satisfaction with a smart-phone self-management support programme to support the self-monitoring of blood pressure, medications, symptom recognition and biochemistry. ${ }^{40}$ In contrast, another study found that text-message-based interventions were the least preferred telehealth intervention for medication monitoring by CKD participants, compared with web-based or personal digital assistant-based applications. ${ }^{41}$ The Effects of Sodium Modification on Outcome (ESMO) study, a 3-month self-management intervention in 138 adults with CKD which provided one-to-one sessions and telephone support, demonstrated relatively high (63\%) satisfaction from participants. It has been postulated that a key factor for the high acceptability of the ESMO intervention was the patient-engagement used in the design of the trial. ${ }^{42}$ This was an approach also taken in the ENTICE-CKD study. We have previously found that patients with CKD have been confused by dietary advice and need more frequent contact to support dietary change. ${ }^{10}$ They were willing to participate in telephone calls and receive text messages, as these were viewed within their comfort zone and levels of digital literacy, ${ }^{10}$ but also raised concerns about the credibility, safety and lack of personalisation in mobile apps and internet modalities. The ENTICE-CKD programme was developed from the key results in this focus group study which assured a patient-centred approach. ${ }^{43}$

Previous thematic synthesis has shown that people with CKD experience many challenges in relation to achieving their dietary and fluid recommendations. People express a preference for regular coaching, feedback and monitoring to help them understand dietary information and become confident in their ability to self-monitor and manage such changes. ${ }^{8}$ The ENTICE-CKD programme was designed to foster incremental dietary advice, with each individual call being dedicated to a separate topic. Each call was also tailored and flexible to participants' goals for dietary change. These attributes may also help explain the difference observed in the acceptability compared with the non-tailored education only (control) intervention.

There are limitations to this study. As we had a $35 \%$ recruitment rate, the feasibility and acceptability only relate to the participants enrolled in this pilot, thus the feasibility for the uptake of the programme and its generalisability in clinical practice are unknown. Furthermore, the baseline health literacy was 'good' in over $90 \%$ of our participants which is likely greater than the health literacy of the wider CKD population. ${ }^{44}$ While other demographics of the people who participated in the ENTICE-CKD study were broadly representative of the CKD demographic reported in international comparisons, ${ }^{45-47}$ we note that previous work has shown that approximately $20 \%-25 \%$ have low health literacy ${ }^{48}$ while only $10 \%$ of our study's participants had low health literacy. We speculate that it is possible that our estimate of health literacy may be inflated due to the singleitem questionnaire having poorer sensitivity for people with marginal reading ability. ${ }^{49}$ Future studies should consider the use of a skill-based health literacy questionnaire, such as the Newest Vital Sign, which might better detect poor levels of health literacy in this population. ${ }^{50}$ We also acknowledge that we captured the individual participant adherence to the intervention using qualitative methods rather than validated surveys. However, given the primary outcome of feasibility, qualitative methods were used to minimise the over-use of self-report surveys and participant burden and this was an exploratory measure of intervention adherence only. Using this method, we were able to capture to reasons for adherence (and non-adherence). We also did not recruit children into the ENTICE-CKD study, so our results are not generalisable to children with CKD. Finally, we did not interview participants in the non-tailored education-only (control) group, and thus could not ascertain the reasons for lower acceptability compared with the intervention group. 
There are several adaptations which should be considered for a future trial based on the findings of this feasibility and acceptability study. First, the generalisability of the study sample could be improved by recruiting participants from primary care (including general practices) and public and private nephrology units. This may improve the recruitment rate, targeting people who are potentially more motivated to change their diets compared with those who have been in the nephrology service for many years. There is also more opportunity for people to consult with a dietitian in specialised nephrology services, evident by $6 \%$ of people who declined to participate doing so because they were already seeing a dietitian. Second, the number and structure of the coaching calls could be modified. All participants who completed call 1 went on to complete at least four calls, however reasons for missing the final two calls did vary and these calls were most commonly used for check-in and review of participant goals only. This could therefore be done at the participant's discretion and to give participants more flexibility which was a key reason for the ENTICE-CKD programme's acceptability. Lastly, due to the unexpectedly large volume of over 1000 'unrecognised' text messages sent by participants, a larger trial would be required to adapt the programme to provide an automated response in these instances.

In conclusion, the ENTICE-CKD dietary coaching programme is a feasible and acceptable intervention for adults with stage 3-4 CKD. The programme facilitated self-monitoring and encouraged the adoption of goal setting throughout the intensive coaching period. Findings from this study are promising for the use of telehealth to modify dietary practices in future clinical practice and research. However, longer-term studies are needed to determine the safety, clinical effectiveness and sustainability before the wider implementation of the ENTICE-CKD programme is appropriate. This process evaluation can be used by clinicians to inform frequent and structured contact through telephone-based and text-message platforms to support the complex dietary self-management required for people with CKD.

\section{Author affiliations}

${ }^{1}$ Faculty of Health Science and Medicine, Bond University, Robina, Queensland,

Australia

${ }^{2}$ Department of Nutrition and Dietetics, Princess Alexandra Hospital, Woolloongabba, Queensland, Australia

${ }^{3}$ Centre for Research in Evidence Based Practice, Faculty of Health Sciences and

Medicine, Bond University, Gold Coast, Queensland, Australia

${ }^{4}$ College of Medicine and Public Health, Flinders University, Adelaide, South

Australia, Australia

${ }^{5}$ Sydney School of Public Health, The University of Sydney, Adelaide, South Australia, Australia

${ }^{6}$ Centre for Kidney Research, The Children's Hospital at Westmead, Westmead, New South Wales, Australia

${ }^{7}$ Cancer Prevention Research Centre, School of Public Health, The University of Queensland, Herston, Queensland, Australia

${ }^{8}$ Department of Nephrology, Princess Alexandra Hospital, Brisbane, Queensland, Australia

${ }^{9}$ Centre for Kidney Disease Research, University of Queensland, Brisbane,

Queensland, Australia

${ }^{10}$ Translational Research Institute, Brisbane, Queensland, Australia
${ }^{11}$ Department of Medicine, University of Otago Christchurch, Christchurch, New Zealand

Contributors JTK wrote the first draft of the manuscript and takes responsibility for the integrity of the data. JTK, KLC, DWJ, MR and SP assisted in the conceptualisation of the trial design. MMW and DPR were responsible for the qualitative data collection and analysis, assisted in the conceptualisation of the qualitative research methods. MMW wrote the qualitative results section of the manuscript. JTK and MC designed the intervention materials and were responsible for the management of the trial at their respective sites. TH, JC and AT provided methodological expertise and revised drafts of the manuscript. All authors contributed to revisions of the manuscript and approved the final version for submission. JTK is the guarantor and affirms that the manuscript is an honest, accurate and transparent account of the study being reported.

Funding This study was supported by a research support grant awarded by Kidney Health Australia via Australasian Kidney Trial Network (AKTN), and a ViceChancellors Research Award through Bond University.

Competing interests None declared.

Patient consent for publication Not required.

Ethics approval This trial was approved by the Metro South Health Service District Human Research Ethics Committee (EC00167) and Bond University Human Research Ethics Committee (EC00357).

Provenance and peer review Not commissioned; externally peer reviewed. Data sharing statement № additional data available.

Open access This is an open access article distributed in accordance with the Creative Commons Attribution Non Commercial (CC BY-NC 4.0) license, which permits others to distribute, remix, adapt, build upon this work non-commercially, and license their derivative works on different terms, provided the original work is properly cited, appropriate credit is given, any changes made indicated, and the use is non-commercial. See: http://creativecommons.org/licenses/by-nc/4.0/.

\section{REFERENCES}

1. Bello AK, Levin A, Tonelli M, et al. Assessment of global kidney health care status. JAMA 2017;317:1864-81.

2. Chen T, Harris DC. Challenges of chronic kidney disease prevention. Med J Aust 2015;203:209-10.

3. Steiber AL. Chronic kidney disease: considerations for nutrition interventions. JPEN J Parenter Enteral Nutr 2014;38:418-26.

4. Ash S, Campbell KL, Bogard J, et al. Nutrition prescription to achieve positive outcomes in chronic kidney disease: a systematic review. Nutrients 2014;6:416-51.

5. Kelly JT, Rossi M, Johnson DW, et al. Beyond sodium, phosphate and potassium: potential dietary interventions in kidney disease. Semin Dial 2017;30:197-202.

6. Kelly JT, Palmer SC, Wai SN, et al. Healthy dietary patterns and risk of mortality and esrd in ckd: a meta-analysis of cohort studies. Clin $J$ Am Soc Nephrol 2017;12:272-9.

7. Wai SN, Kelly JT, Johnson DW, et al. Dietary patterns and clinical outcomes in chronic kidney disease: the CKD.QLD Nutrition Study. $J$ Ren Nutr 2017;27:175-82.

8. Palmer SC, Hanson CS, Craig JC, et al. Dietary and fluid restrictions in CKD: a thematic synthesis of patient views from qualitative studies. Am J Kidney Dis 2015;65:559-73.

9. Hand RK, Steiber A, Burrowes J. Renal dietitians lack time and resources to follow the NKF KDOQI guidelines for frequency and method of diet assessment: results of a survey. $J$ Ren Nutr 2013;23:445-9.

10. Kelly JT, Campbell KL, Hoffmann T, et al. Patient Experiences of Dietary Management in Chronic Kidney Disease: A Focus Group Study. J Ren Nutr 2018;28:393-402.

11. Kelly JT, Reidlinger DP, Hoffmann TC, et al. Telehealth methods to deliver dietary interventions in adults with chronic disease: a systematic review and meta-analysis. Am J Clin Nutr 2016;104:1693-702.

12. Beall RF, Baskerville N, Golfam M, et al. Modes of delivery in preventive intervention studies: a rapid review. Eur J Clin Invest 2014;44:688-96.

13. Desroches S, Lapointe A, Ratté $S$, et al. Interventions to enhance adherence to dietary advice for preventing and managing chronic diseases in adults. Cochrane Database Syst Rev 2013;2:Cd008722. 
14. Direito A, Dale LP, Shields E, et al. Do physical activity and dietary smartphone applications incorporate evidence-based behaviour change techniques? BMC Public Health 2014;14:646.

15. Goode AD, Reeves MM, Eakin EG. Telephone-delivered interventions for physical activity and dietary behavior change: an updated systematic review. Am J Prev Med 2012;42:81.

16. Norman GJ, Zabinski MF, Adams MA, et al. A review of eHealth interventions for physical activity and dietary behavior change. Am J Prev Med 2007;33:336-45. e16.

17. Spark LC, Fjeldsoe BS, Eakin EG, et al. Efficacy of a Text MessageDelivered Extended Contact Intervention on Maintenance of Weight Loss, Physical Activity, and Dietary Behavior Change. JMIR Mhealth Uhealth 2015;3:e88.

18. Fjeldsoe B, Neuhaus M, Winkler E, et al. Systematic review of maintenance of behavior change following physical activity and dietary interventions. Health Psychol 2011;30:99-109.

19. Hall AK, Cole-Lewis H, Bernhardt JM. Mobile text messaging for health: a systematic review of reviews. Annu Rev Public Health 2015;36:393-415.

20. Chow CK, Redfern J, Hillis GS, et al. Effect of lifestyle-focused text messaging on risk factor modification in patients with coronary heart disease: a randomized clinical trial. JAMA 2015;314:1255-63.

21. Bobrow K, Farmer AJ, Springer D, et al. Mobile Phone Text Messages to Support Treatment Adherence in Adults With High Blood Pressure (SMS-Text Adherence Support [StAR]): A SingleBlind, Randomized Trial. Circulation 2016;133.

22. Eldridge SM, Chan CL, Campbell MJ, et al. CONSORT 2010 statement: extension to randomised pilot and feasibility trials. Pilot Feasibility Stud 2016;2:64.

23. Bandura A. Social foundations of thought and action: A social cognitive theory: Prentice-Hall, Inc, 1986

24. NHMRC. Australian Dietary Guidelines. Canberra: National Health and Medical Research Council, Department of Health and Ageing, 2013.

25. Hoffmann TC, Glasziou PP, Boutron I, et al. Better reporting of interventions: template for intervention description and replication (TIDieR) checklist and guide. BMJ 2014;348:g1687.

26. Australian Government Department of Health. Australia's physical activity and sedentary behaviour guidelines for adults (18-64 years): Commonwealth of Australia, 2014.

27. Whitlock EP, Orleans CT, Pender N, et al. Evaluating primary care behavioral counseling interventions: an evidence-based approach Am J Prev Med 2002;22:267-84.

28. Microsoft Excel [program, 2011. 14.4.5 version.

29. Fjeldsoe BS, Goode AD, Phongsavan P, et al. Evaluating the maintenance of lifestyle changes in a randomized controlled trial of the 'get healthy, stay healthy' program. JMIR Mhealth Uhealth 2016; $4: \mathrm{e} 42$

30. ABS. Census of population and housing: Socio-economic indexes for areas (SEIFA), Australia, 2011: Australian Bureau of Statistics, 2015.

31. Morris NS, MacLean CD, Chew LD, et al. The single item literacy screener: evaluation of a brief instrument to identify limited reading ability. BMC Fam Pract 2006;7:21.

32. Lancaster GA, Dodd S, Williamson PR. Design and analysis of pilot studies: recommendations for good practice. $J$ Eval Clin Pract 2004;10:307-12.
33. Vaismoradi M, Turunen $\mathrm{H}$, Bondas T. Content analysis and thematic analysis: Implications for conducting a qualitative descriptive study. Nurs Health Sci 2013;15:398-405.

34. Microsoft Word [program]. 14.4 .5 version, 2011.

35. Welch JL, Johnson M, Zimmerman L, et al. Self-management interventions in stages 1 to 4 chronic kidney disease: an integrative review. West J Nurs Res 2015;37:652-78.

36. Whelan ME, Goode AD, Eakin EG, et al. Feasibility, effectiveness and cost-effectiveness of a telephone-based weight loss program delivered via a hospital outpatient setting. Trans/ Behav Med 2016;6:386-95

37. Partridge SR, Allman-Farinelli M, McGeechan K, et al. Process evaluation of TXT2BFiT: a multi-component mHealth randomised controlled trial to prevent weight gain in young adults. Int J Behav Nutr Phys Act 2016;13:7.

38. Blakeman T, Blickem C, Kennedy A, et al. Effect of information and telephone-guided access to community support for people with chronic kidney disease: randomised controlled trial. PLoS One 2014;9:e109135

39. Tuot DS, Boulware LE. Telehealth applications to enhance CKD knowledge and awareness among patients and providers. Adv Chronic Kidney Dis 2017;24:39-45.

40. Ong SW, Jassal SV, Miller JA, et al. Integrating a smartphone-based self-management system into usual care of advanced CKD. Clin J Am Soc Nephrol 2016;11:1054-62.

41. Diamantidis CJ, Zuckerman M, Fink W, et al. Usability testing and acceptance of an electronic medication inquiry system for CKD patients. Am J Kidney Dis 2013;61:644-6.

42. Campbell KL, Palmer SC, Johnson DW. Improving nutrition research in nephrology: an appetite for change. Am J Kidney Dis 2017;69:558-60.

43. Domecq JP, Prutsky G, Elraiyah T, et al. Patient engagement in research: a systematic review. BMC Health Serv Res 2014;14:89.

44. Wright Nunes JA, Wallston KA, Eden SK, et al. Associations among perceived and objective disease knowledge and satisfaction with physician communication in patients with chronic kidney disease. Kidney Int 2011;80:1344-51.

45. Hill NR, Fatoba ST, Oke JL, et al. Global prevalence of chronic kidney disease - a systematic review and meta-analysis. PLoS One 2016;11:e0158765.

46. Mahmood U, Healy HG, Kark A, et al. Spectrum (characteristics) of patients with chronic kidney disease (CKD) with increasing age in a major metropolitan renal service. BMC Nephrol 2017;18:372.

47. Yang W, Xie D, Anderson AH, et al. Association of kidney disease outcomes with risk factors for CKD: findings from the Chronic Renal Insufficiency Cohort (CRIC) study. Am J Kidney Dis 2014;63:236-43.

48. Fraser SD, Roderick PJ, Casey M, et al. Prevalence and associations of limited health literacy in chronic kidney disease: a systematic review. Nephrol Dial Transplant 2013;28:129-37.

49. Morris NS, MacLean CD, Chew LD, et al. The Single Item Literacy Screener: evaluation of a brief instrument to identify limited reading ability. BMC Fam Pract 2006;7:21.

50. Weiss BD, Mays MZ, Martz W, et al. Quick assessment of literacy in primary care: the newest vital sign. Ann Fam Med 2005;3:514-22. 